Pediat. Res. 1: 372-385 (1967)

Phenylketonuria indole kynurenine phenylalanine tryptophan mental retardation

\title{
Tryptophan Oxidation in Phenylketonuria
}

\author{
J.A.Anderson ${ }^{[38]}$, H.Bruhl, A.J. Mrahaels and Doris Doeden \\ Department of Pediatrics, College of Medical Sciences, University of Minnesota, Minneapolis, \\ and Faribault State School and Hospital, Faribault, Minnesota, USA
}

\begin{abstract}
Extract
Tryptophan oxidation was evaluated in 6 institutionalized phenylketonuric subjects (age range 5-13 years) by measuring the urinary excretion of oxidation metabolites following administration of $\mathrm{L}$ tryptophan loads by mouth. Observations were made when subjects were on a general diet, a low. phenylalanine diet and a low-phenylalanine diet containing added L-phenylalanine. At some time during each dietary regime, the subjects also received tryptophan by mouth, $100 \mathrm{mg} / \mathrm{kg}$ body weight. Measurement was made in urine of: 1. 3-hydroxykynurenine; 2. kynurenine; 3. acetyl kynurenine; 4. 3-hydroxyanthranilic acid; 5 . xanthurenic acid; 6 . kynurenic acid; 7. anthranilic acid; 8. 3-indoleacetic acid; 9. o-hydroxyphenylacetic acid; and 10. phenylpyruvic acid. Excretion of tryptophankynurenine metabolites (products 1-9) amounted to $3.78 \mu \mathrm{moles} / \mathrm{kg} / 24 \mathrm{~h}$ and was similar to that of control subjects while on all diets. With tryptophan loading on normal diets, excretion of metabolic products was lower than for control subjects. With tryptophan loading on low-phenylalanine diets, the excretion of tryptophan metabolites was 2-3 times higher than the mean value of $22.7 \mu$ moles $/ \mathrm{kg} /$ $24 \mathrm{~h}$ observed in normal subjects. The response to loading when the diet contained added phenylalanine was variable, since two patients excreted normal amounts of metabolites and three excreted amounts several times greater than the controls. Excretion of indole and 3-indoleacetic acid was abnormally elevated with and without tryptophan loading on normal diets and when phenylalanine was added to the low-phenylalanine diets. Values in $\mu$ moles $/ \mathrm{kg} / 24 \mathrm{~h}$ were $: 3$-indoleacetic acid 1.7 (normal) ; 2.5-9.4 (PKU); indican 6.24 (normal); 14.9-24.6 (PKU).
\end{abstract}

Speculation

Limited metabolism of tryptophan may be of importance to central nervous system development and function in certain phenylketonuric children. Malabsorption of tryptophan coupled with an inhibition of the tryptophan-kynurenine synthesis pathway for nicotinic acid could deprive the central nervous system of important cofactors essential to central nervous system metabolism. The subtle effects of secondary disturbances on enzymatic pathways for tryptophan, tyrosine and other amino acids may be more important than the direct influence of the metabolites of phenylalanine on the central nervous system in phenylketonuric subjects. 


\section{Introduction}

Interference with the metabolism of tryptophan along several metabolic pathways has been noted in phenylketonuria. Limitation in the rate of synthesis of serotonin $[4,27]$ increased formation of indole compounds $[3,30]$ and decreased rate of oxidation of tryptophan to kynurenine have been reported [31]. Certain clinical studies, in part supported by in vitro observations, suggest that the basic derangements in the abovemetabolic abnormalities are: 1. competitive inhibition of tryptophan hydroxylase by phenylalanine and certain of its metabolites $[10,28] ; 2$. faulty intestinal absorption of tryptophan resulting in increased enteric bacterial metabolic sources of indole compounds; 3 . inefficient endogenous metabolism of indoles $[3,5,6]$; and 4 . an inhibition of the activity of the hepatic tryptophan pyrrolase required for the conversion of tryptophan to formyl-kynurenine [24, 31].

This present study is primarily concerned with the third disturbance, the oxidation of tryptophan to kynurenine and further kynurenine pathway metabolites in phenylketonuric subjects. Inhibition of the oxidative pathway for tryptophan has theoretical importance in phenylketonuria since endogenous nicotinic acid is produced along this metabolic pathway. An associated limitation in absorption of tryptophan from the intestinal tract may potentially further reduce endogenous nicotinic acid production.

It has been suggested that phenylpyruvic and orthohydroxyphenylacetic acids, indole and skatole, all produced excessively in phenylketonuria, may individually as well as collectively partially inhibit tryptophan pyrrolase activity in vitro [6]. Evidence of in vivo inhibition has been provided by a limited increase in the excretion of tryptophan-kynurenine metabolites following an oral load of L-tryptophan [31].

In the present study, the basis for interpretation of observed changes in the urinary excretion of kynurenine and the further oxidative pathway metabolites resides in the observation that a direct correlation exists between the activity of hepatic tryptophan pyrrolase and the amount of urinary kynurenine excreted [1]. Tryptophan pyrrolase is required for the conversion of tryptophan to formylkynurenine, the first metabolic step in this pathway [15]. Tryptophan is the specific substrate inducer which evokes the formation of and stabilizes the active reduced form of holotryptophan pyrrolase [16]. Hydrocortisone is also an inducer acting by increasing the rate of synthesis of the apotryptophan pyrrolase protein moeity $[11,17]$. Thus, factors which increase the rate of production of adrenocorticotrophic hormone and hence the outflow of hydrocortisone may indirectly modify tryptophan pyrrolase activity.
In the present study, the changes in the urinary excretion of tryptophan kynurenine metabolites have been observed in phenylketonuric subjects during various dietary programs in which the L-phenylalanine content was modified.

\section{Study Procedures}

Six institutionalized phenylketonuric children were studied. The age, sex, body weight and reference number to the data tables are as follows: 1.5 years, male, $19 \mathrm{~kg} ; 2.8$ years, female, $22 \mathrm{~kg} ; 3.11$ years, male, $25 \mathrm{~kg}$; 4.7 years, male, $26 \mathrm{~kg} ; 5.11$ years, female, $28 \mathrm{~kg}$; and 6.13 years, female, $34 \mathrm{~kg}$. The subjects were given oral tryptophan loads (100 mg/kg body weight) during the course of the three diet programs; a general diet, a low-phenylalanine diet and a lowphenylalanine diet to which L-phenylalanine $100 \mathrm{mg}$ / $\mathrm{kg}$ body weight had been added. No supplementary natural foods were used during the low-phenylalanine diet periods. The low-phenylalanine diet used contained $0.8 \%$ L-tyrosine and $0.2 \%$ L-tryptophan ${ }^{1}$. Hence $1.76 \mathrm{~g}$ of tyrosine per 1000 calories was available on the low-phenylalanine diet compared with about $1.0 \mathrm{~g}$ available on the regular diet.

Tryptophan loading studies were done in four of the subjects on the general diet prior to starting the low-phenylalanine diet program. After several weeks on the low-phenylalanine diet, the tryptophan loading studies were repeated. Following this, L-phenylalanine was added to the low-phenylalanine diet for a period of six to seven days and the loading studies were again done on the last day. Two subjects were on the lowphenylalanine diet for many months, and the tryptophan loading studies were first done on the low-phenylalanine diet and the low-phenylalanine plus phenylalanine diet. They were then placed on the general diet and tryptophan loading studies were repeated. Twenty-four-hour urine collections were made on the day prior to and on the day of the oral tryptophan loading tests. A retention catheter was used in the female subjects to collect specimens during the control and the loading day to assure complete collection. Careful clinical and laboratory evaluation following catheterization failed to reveal evidence of infection in the genito-urinary tract. No medications were given during the study periods.

Acetylkynurenine, kynurenine, anthranilic acid and anthranilic glucuronide were determined by the elution column chromatographic methods of BRown and PRICE [8]. The columns were prepared with a Dowex

\footnotetext{
${ }^{1}$ Low-phenylalanine diet, Lofanolac ${ }^{\circledR}$, Mead Johnson Company, Evansville, Indiana.
} 
$50 \mathrm{~W}-\mathrm{X} 12$ (200 to $400 \mathrm{mesh}$ ) and were first washed with $80 \mathrm{ml}$ of $8.0 \mathrm{~N}$ hydrochloric acid followed by 200 $\mathrm{ml}$ of distilled water and then $25 \mathrm{ml}$ of $0.1 \mathrm{~N}$ hydrochloric acid as recommended by TOMPSETT [32]. All urine samples were analyzed in duplicate by diluting 10 to $30 \mathrm{ml}$ of the pretryptophan urine specimens and 2 to $10 \mathrm{ml}$ of post load samples to a volume of $40 \mathrm{ml}$ and a final hydrochloric acid concentration of $0.10 \mathrm{~N}$. In each instance, a recovery control was also prepared by adding 250 to $500 \mu \mathrm{g}$ of each metabolite to a similar volume of urine specimen. Recoveries of the various metabolites were between 85 and $105 \%$. The colorimetric determination of the diazotizable amines present in the various fractions was performed according to the method of Brown and PRICE [8].

3-hydroxykynurenine was determined as described by BRown [9]. 3-hydroxyanthranilic acid was determined by a modification of the method described by ToMpsetr [32]. Instead of using 2,6-dichloroquinone chlorimide to develop color, the determination was carried out with the color reaction that develops after the addition of $0.25 \%$ sodium nitrite and $10 \%$ ammonium sulfamate as described in the method for 3-hydroxykynurenine. The results agreed closely with the colorimetric method of TOMPSETT. Kynurenic and xanthurenic acids were separated by elution chromatography with Dowex $50 \mathrm{~W}-\mathrm{X} 12$ and measured in an Aminco-Bowman spectrofluorometer using the method of SAtoH and Price [29].

3 -indoleacetic acid was determined by the method of Weissbach, King, SJoerdsma and Udenfriend [35]. The urine sample was hydrolyzed first, so that the determination represents the free and conjugated 3indoleacetic acids and may also contain indoleacetamide and indolelactic acid. Urinary indican was determined according to the method of MeIkLeJohn and CoHen [22].

The ortho-hydroxyphenylacetic acid was measured both by a biochemical method described by ANDERson, Fisch, Mrrler and Doeden [2] and by gas chromatography using the method described by Willuams and SwEELEy [36]. The results reported here are the values obtained by either method, both providing above $85 \%$ recovery of added orthohydroxyphenylacetic acid from urine.

Phenylpyruvic acid in the urine was measured by the method of KROPP and LANG [19].

Serum phenylalanine concentration was measured by the method of MaCaman and RoBins [21] and serum tyrosine concentration by the method of WAALKES and UDENFRIEND [33].
Results

The urinary excretion of tryptophan-kynurenine meta. bolites in five of the six subjects observed while on the general diet, low-phenylalanine diet containing added L-phenylalanine, and the responses obtained on each of the diet periods to oral L-tryptophan loads have been presented in tables I, II and III. The excretion values for control subjects, previously reported from this laboratory [24] are presented at the bottom of each table. The data obtained for Subject 6, a sexually mature female, has been presented separately in table IV with appropriate control values for comparison. The results are reported as $\mu \mathrm{moles} / \mathrm{kg} /$ day unless otherwise specified.

The preloading excretion values for the tryptophankynurenine metabolites (kynurenine plus acetylkynurenine, 3-hydroxykynurenine, 3-hydroxyanthranilic acid, xanthurenic, kynurenic and anthranilic acids) during the general diet, the low-phenylalanine diet and the low-phenylalanine plus L-phenylalanine diet for all of the phenylketonuric subjects were well within the range of $3.78 \pm 1.28$ found for normal subjects (tables I, II and III). Prior to loading, the excretion of the six tryptophan-kynurenine metabolites found in the sexually mature female, Subject 6 , were respectively: 4.60, 3.60 and 3.96. On the general diet, the lowphenylalanine diet and the low-phenylalanine plus phenylalanine diet values were not greatly different from the mean value of $3.1 \pm 1.8$ found for control female subjects (table IV). The relative amount of each of the six metabolites excreted during the preloading periods on the three diet programs was not different from that found for control subjects.

\section{Tryptophan Loading Responses on the General Diet}

The tryptophan loading excretion responses for the six phenylketonuric subjects when on the general diet were significantly less than in control subjects in four of the six phenylketonuric children, Subjects 1, 3 and 4 (table I) and Subject 6 (table IV), and essentially normal in two subjects. This reduced excretion response to tryptophan loading was shared by all six of the metabolites and appeared to be relative to the amount of kynurenine excreted. Subject 2 and Subject 5 had normal loading responses for all kynureninemetabolites; however, Subject 2 exhibited an unusual amount of anthranilic acid and anthranilic glucuronide.

The reduced excretion of the six metabolites following tryptophan loading did not consistently correlate with the amount of phenylpyruvic or ortho-hydroxyphenylacetic acid excreted, or with the serum phenylalanine concentration. Subjects 2 and 5, who had essentially normal tryptophan loading responses, had equally high serum phenylalanine concentrations and 


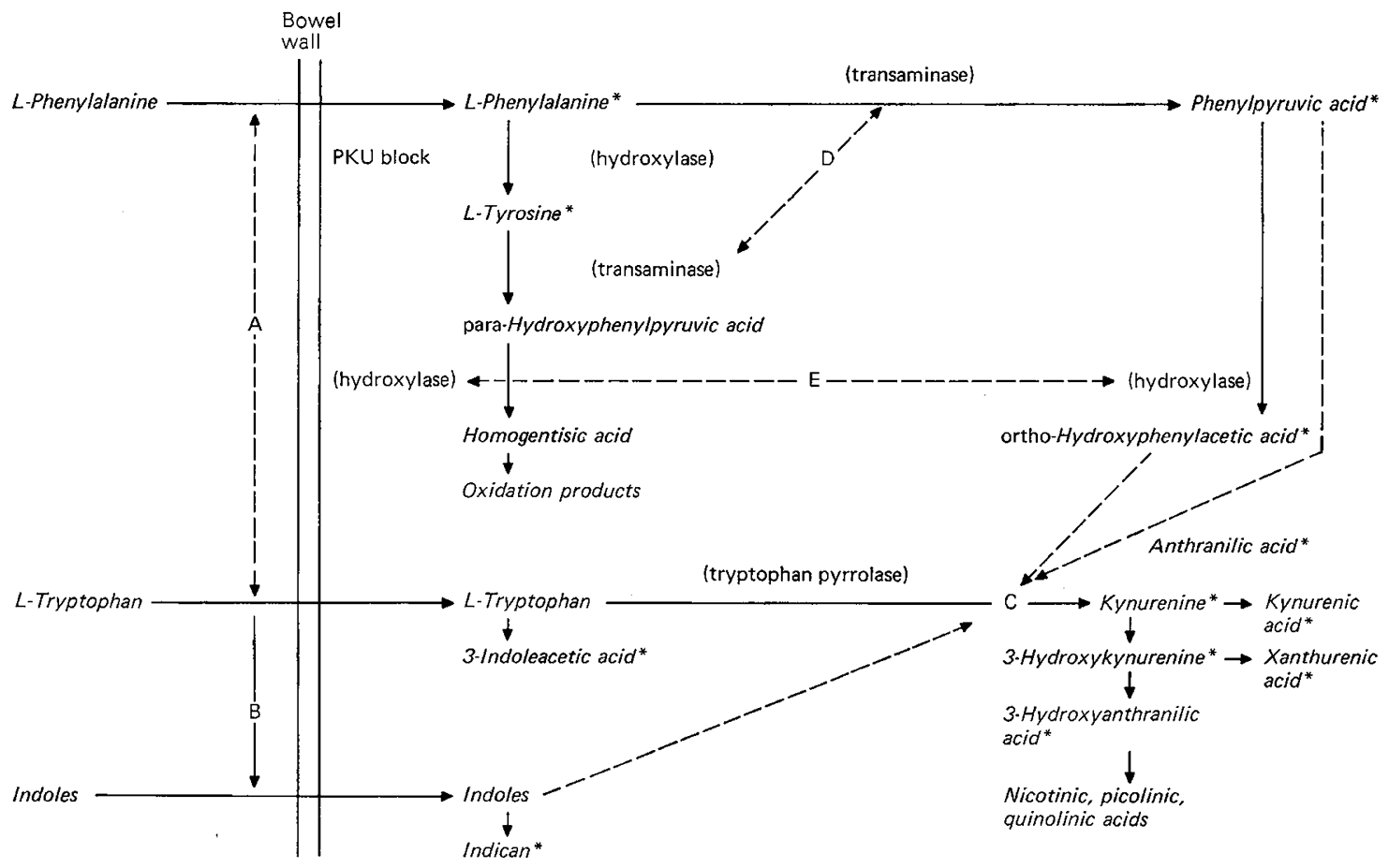

excreted equally large amounts of phenylpyruvic acid when compared with Subjects 1, 3, 4 and 6, who failed to have a normal excretion response to the L-tryptophan load.

Tryptophan Loading Responses on the Low-Phenylalanine Diet During the low-phenylalanine diet period, concentrations of phenylalanine in serum fell to within normal ranges in Subjects 3, 4, 5 and 6; however, these were still slightly elevated in Subjects 1 and 2 at the time that the tryptophan loading studies were done. A corresponding fall in the excretion of phenylpyruvic acid and ortho-hydroxyphenylacetic acid occurred. Tryptophan loading at this time resulted in a great increase in the excretion of the six tryptophan-kynurenine metabolites in five of the six subjects. The total of these six metabolites excreted exceeded by two- to three-fold the mean value of $22.37 \pm 9.6$ found for loaded control subjects (tables II and IV). Subject 4 was of considerable interest. While receiving the general diet, the concentrations of phenylalanine in serum and excretions of phenylpyruvic acid were elevated. Following tryptophan loading (table I), the excretion of tryptophankynurenine metabolites fell from 6.8 to 5.44. Further, when this child was placed on the low-phenylalanine diet, the sum of metabolites excreted on the preloading day was 2.72 and rose in response to tryptophan loading to only 12.5 , about one-half the response anticipated for control subjects.
Fig.1. Schematic representation of the metabolic pathways considered in this study. Blood and urinary metabolites measured, indicated by an asterisk. Theoretical consideration of interrelation of phenylalanine to tryptophan metabolism include: A. competitive absorption at the bowel wall; $\mathrm{B}$. increased bacterial production of indoles from unabsorbed tryptophan; C. inhibition of tryptophan pyrrolase by ortho-hydroxyphenylacetic acid, phenylpyruvic acid and indoles; D. phenylalanine-tyrosine transaminase competition; and E. para-hydroxyphenylpyruvic acid-phenylpyruvic acid hydroxylase competition.

\section{Tryptophan Loading Responses on Low-Phenylalanine Plus L-Phenylalanine Diet}

The incorporation of L-phenylalanine, $100 \mathrm{mg} / \mathrm{kg} /$ day in the low-phenylalanine diet for a period of six to seven days increased the serum phenylalanine concentration to approximately the same high concentrations found when the patients were on the general diet. The serum tyrosine concentrations were also somewhat higher in three of the four subjects studied. In spite of a high serum phenylalanine concentration, the excretion of phenylpyruvic acid increased only 30 to $50 \%$ of the amount excreted when the subjects were on the general diet (table III). In those subjects also excreting large amounts of ortho-hydroxyphenylacetic acid when on the general diet, less excretion was also noted 
376

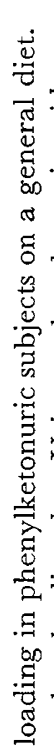

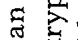

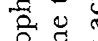

荌

तี.

कo 룰

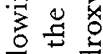

응 홍

च च

융

눙응 중

范苞

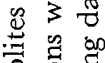

敢.

छ ह

范

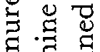

告

क्ष

है

要

รี

要

뙤

$\sim-\frac{2}{0}$

\&

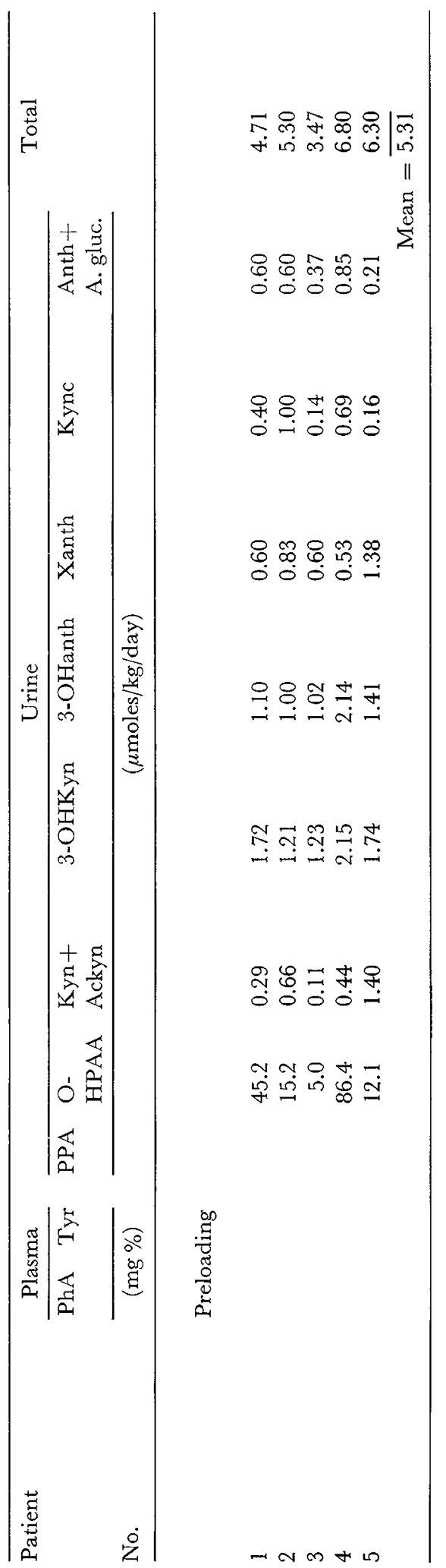

Anderson, Bruhl, Mighaels, Doeden

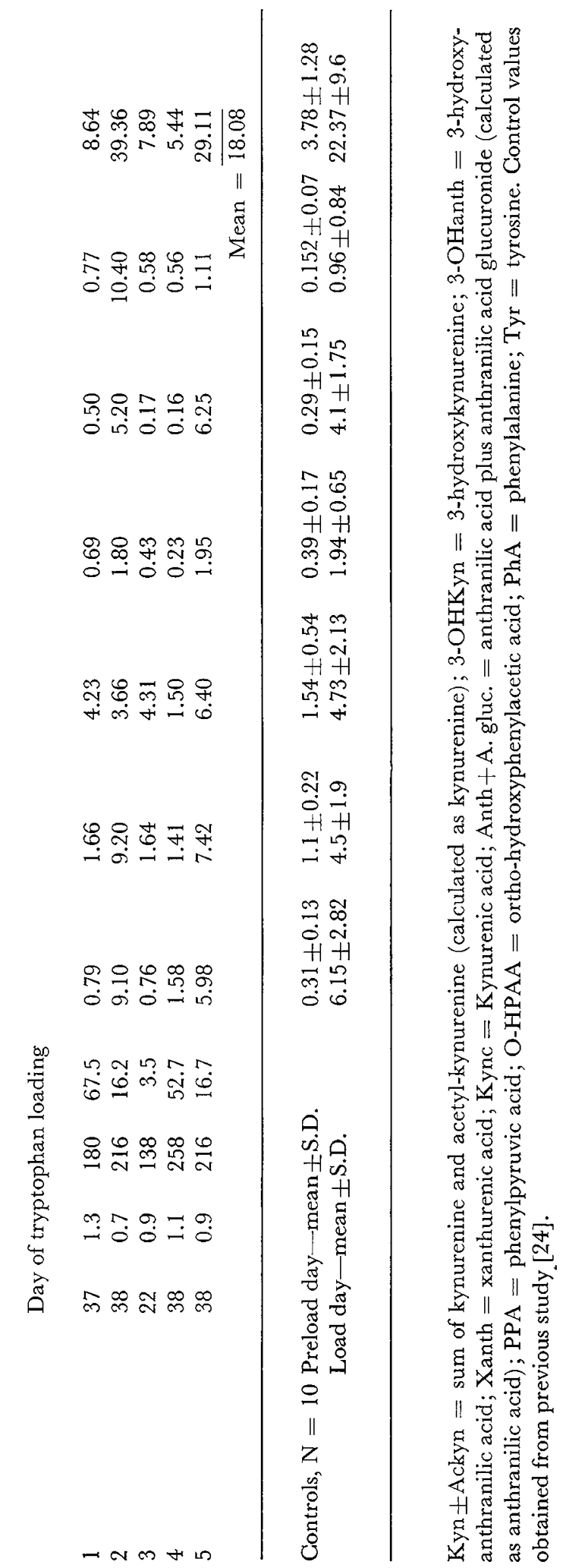


Tryptophan oxidation in phenylketonuria

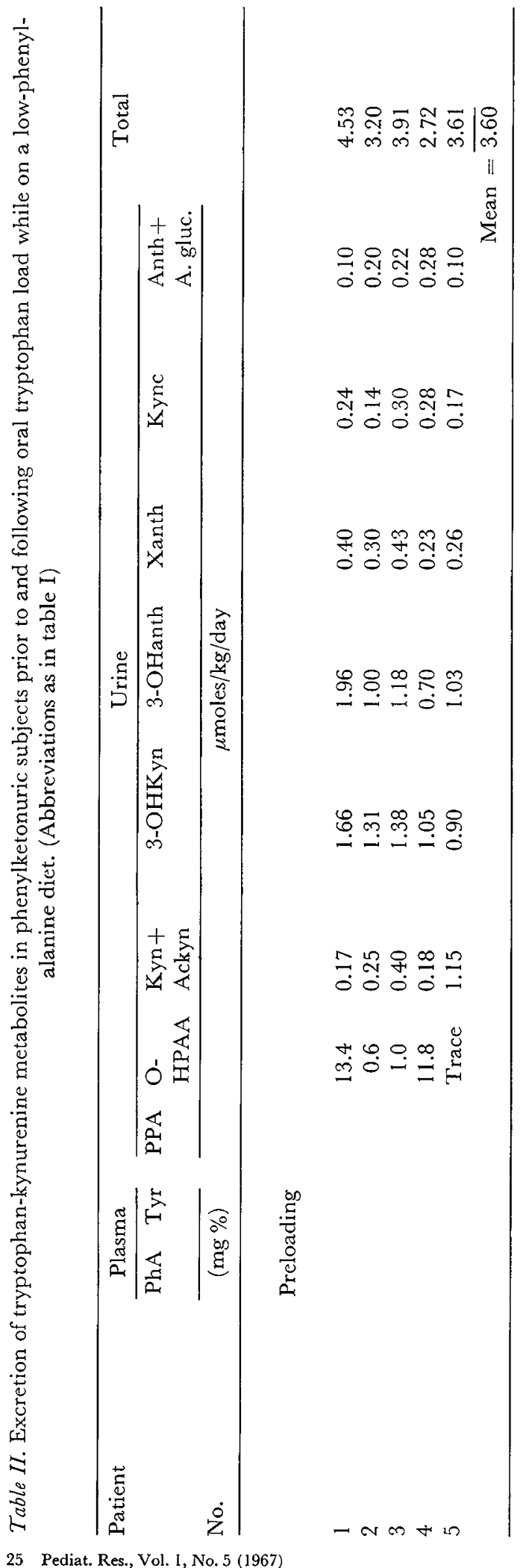

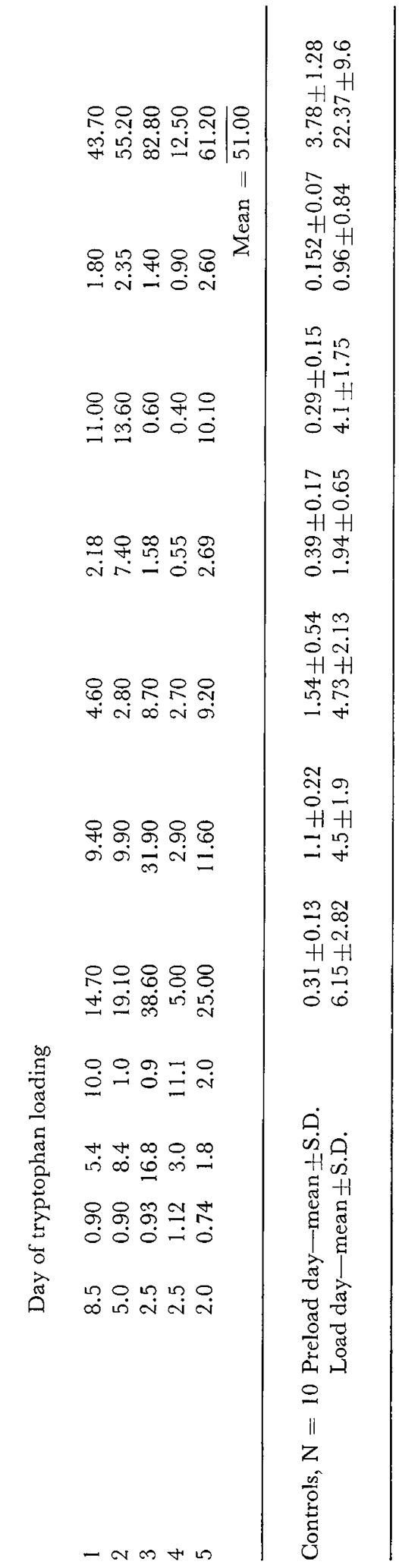

25 Pediat. Res., Vol. I, No. 5 (1967) 


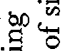

㻤

亮

起

票

की

苛

U.

त

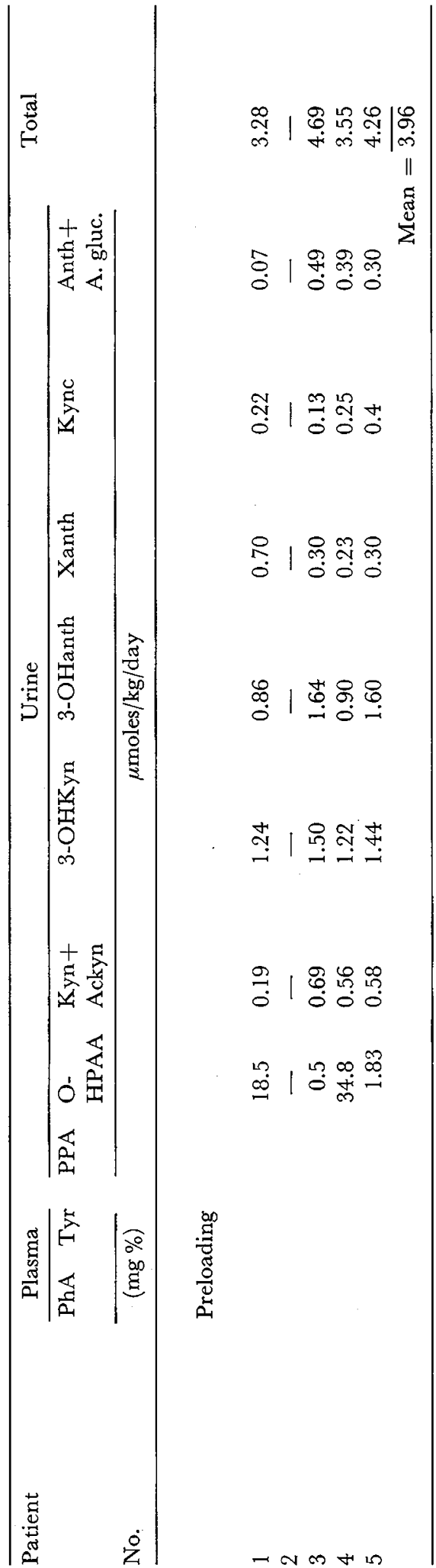

Anderson, Bruhr, Mrahaels, Doeden

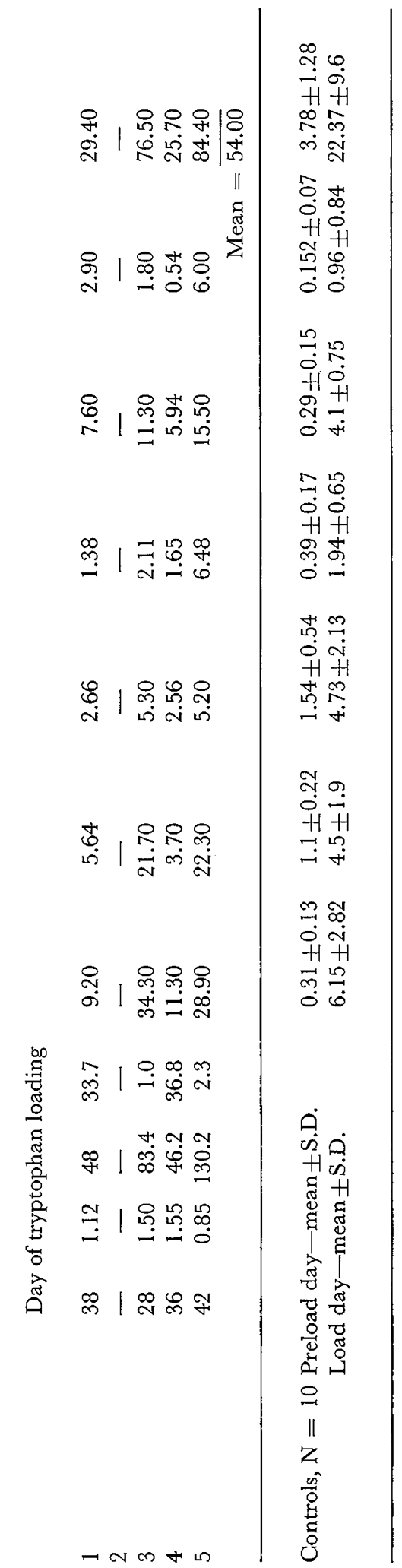


Tryptophan oxidation in phenylketonuria

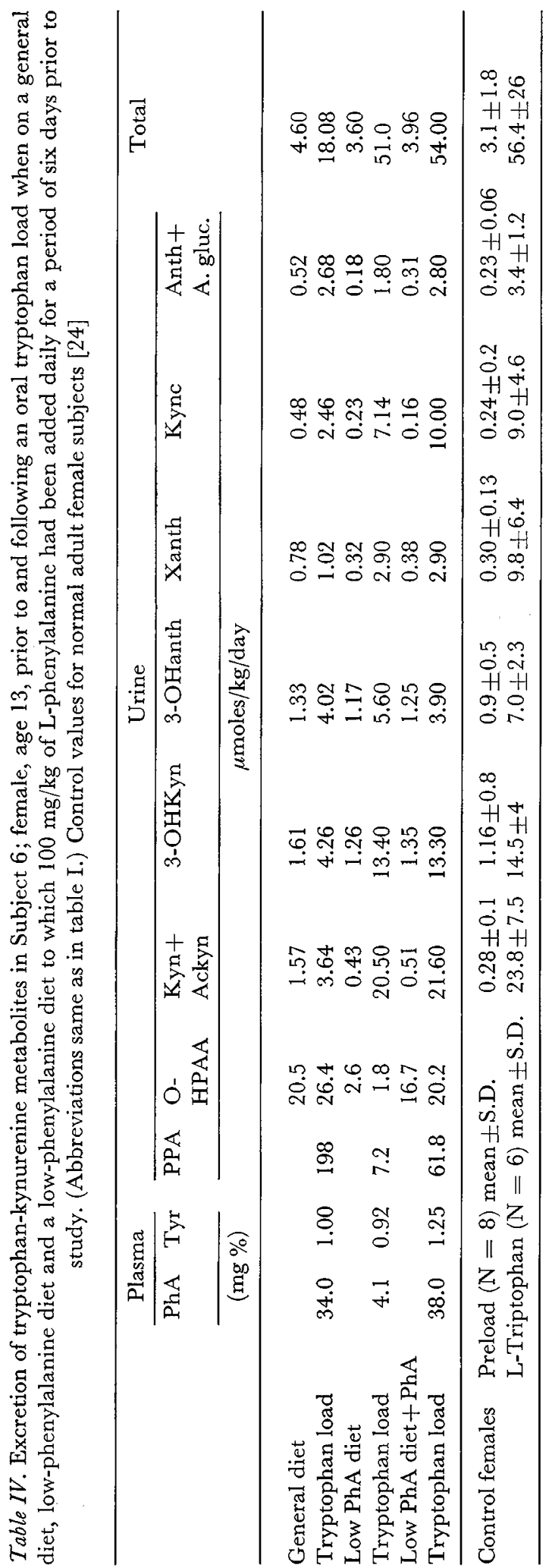

when on the low-phenylalanine plus L-phenylalanine diet. In spite of the presence of a high serum phenylalanine concentration, though less phenylpyruvic acid excretion, oral tryptophan loading still evoked an essentially normal excretion of tryptophan metabolites in two of the subjects, Subjects 1 and 4. However, an excretion response approximately three times greater than the mean observed for tryptophan-loaded control subjects occurred in Subjects 3, 5 and 6 (tables III and IV).

\section{Excretion of 3-Indoleacetic Acid and Indican}

Previous observations in this laboratory indicate that normal children excrete approximately $1.7 \pm 0.75$ $\mu \mathrm{moles} / \mathrm{kg} /$ day of 3 -indoleacetic acid and $6.24 \pm 3.0$ $\mu$ moles $/ \mathrm{kg} /$ day of indican [24]. Further, an oral load of $100 \mathrm{mg} / \mathrm{kg}$ body weight of L-tryptophan is probably completely absorbed as no change in the excretion of indican is observed. Small changes in 3-indoleacetic acid excretion may occur, probably derived in part from endogenous metabolism in tissues [35]. The occurrence of above normal excretion of indican and 3indoleacetic acid prior to loading and an increase in response to an oral tryptophan load may be indicative of faulty absorption of tryptophan [3, 5, 6 and 24]. It was important to evaluate these factors, since preferential shunting of tryptophan to bacterial metabolism may have contributed to the limited absorption of the tryptophan load. Under these circumstances, tryptophan would have been less available for the formation of tryptophan-kynurenine metabolites.

When on the general diet, four of the six subjects excreted two to four times more indican during the preload period ( 14.9 to $24.6 \mu \mathrm{mole} / \mathrm{kg} /$ day) than has been noted for control subjects $(6.24 \pm 3.0$, table V). The excretion in two subjects was within the range found for control subjects. The effect of tryptophan loading on the excretion of indican was variable. No change in two subjects, a slight increase in one subject and a slight fall in two subjects occurred (table V).

The excretion of 3-indoleacetic acid during the preloading general diet period (2.5 to 9.4) was approximately two to four times the value of $1.7 \pm 0.75$ found for control subjects (table V). The administration of the tryptophan load did not produce significant increases in these above normal excretion values except for Subject 5, who excreted $19.5 \mu$ moles $/ \mathrm{kg} /$ day. It should be noted that Subject 5 also had a normal tryptophan-kynurenine excretion response when loaded on a general diet (table I). It appeared that when the subjects were on the general diet and during both the preloading and the loading periods, the urinary excretion of these two metabolites was greater than normal; this reflected to some degree limited absorption of tryptophan at a time when the concentrations of phenylala- 
Table $V$. Urinary excretion of phenylpyruvic acid (PPA), ortho-hydroxyphenylacetic acid (O-HPAA), 3-indoleacetic acid (I-3-AA) and indican prior to and following an oral L-tryptophan load in phenylketonuric subjects on a general diet. Control data [24]

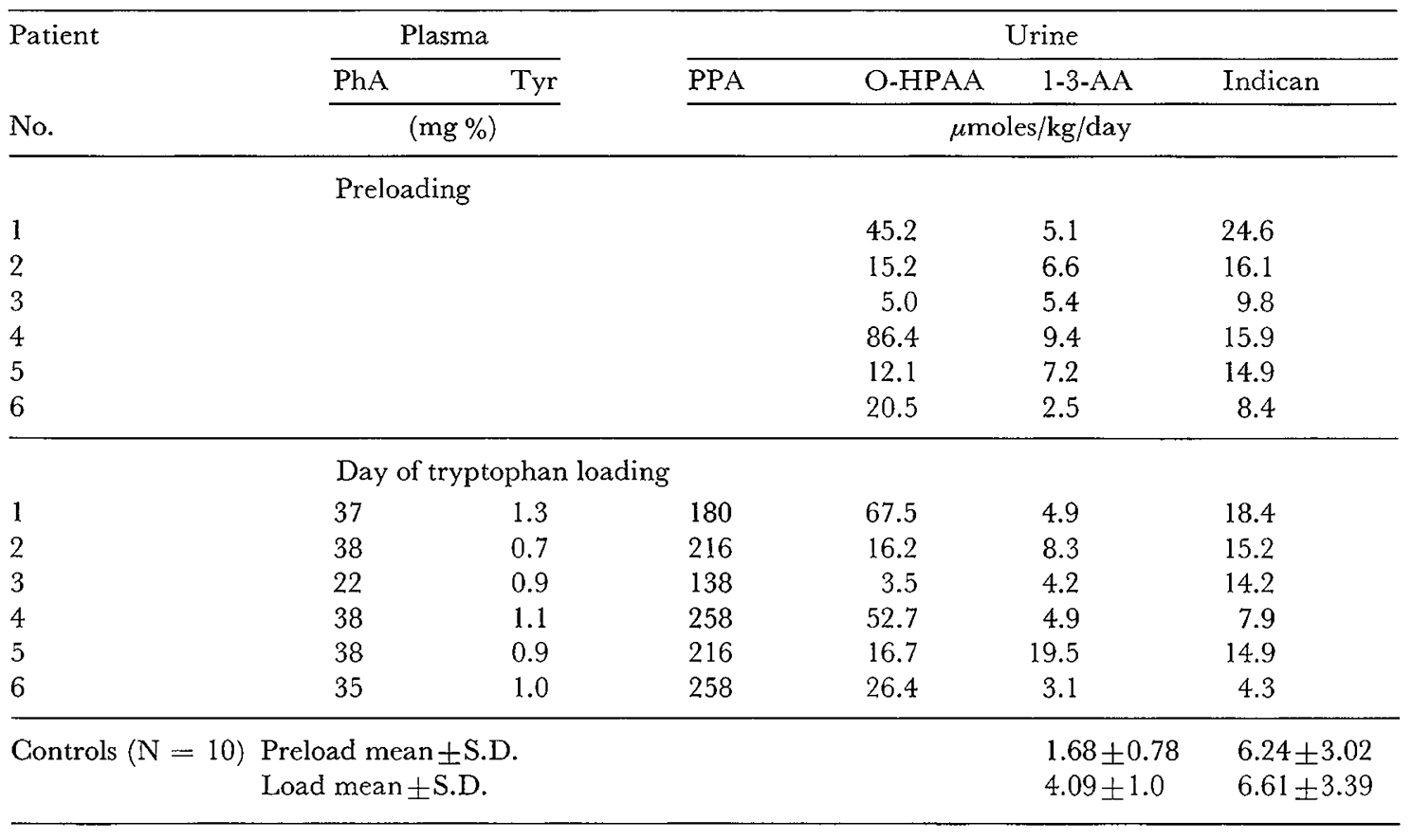

Table VI. Same as table V except subjects had been on a low-phenylalanine diet for at least six days prior to loading studies

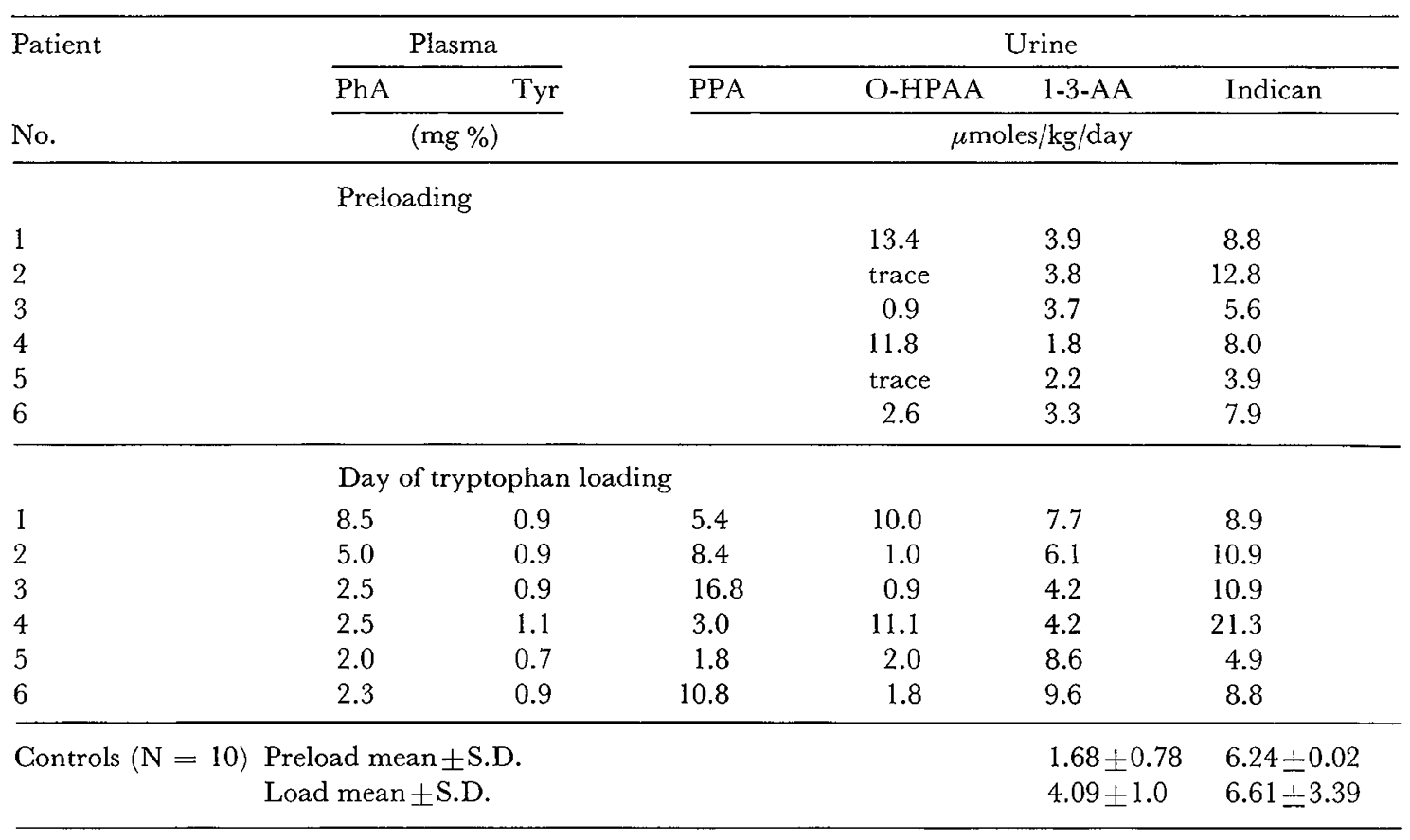


Table VII. Same as table V except subjects had been on a low-phenylalanine diet to which $100 \mathrm{mg} / \mathrm{kg}$ body weight of L-phenylalanine had been added for a period of six days prior to loading studies. Control values [24]

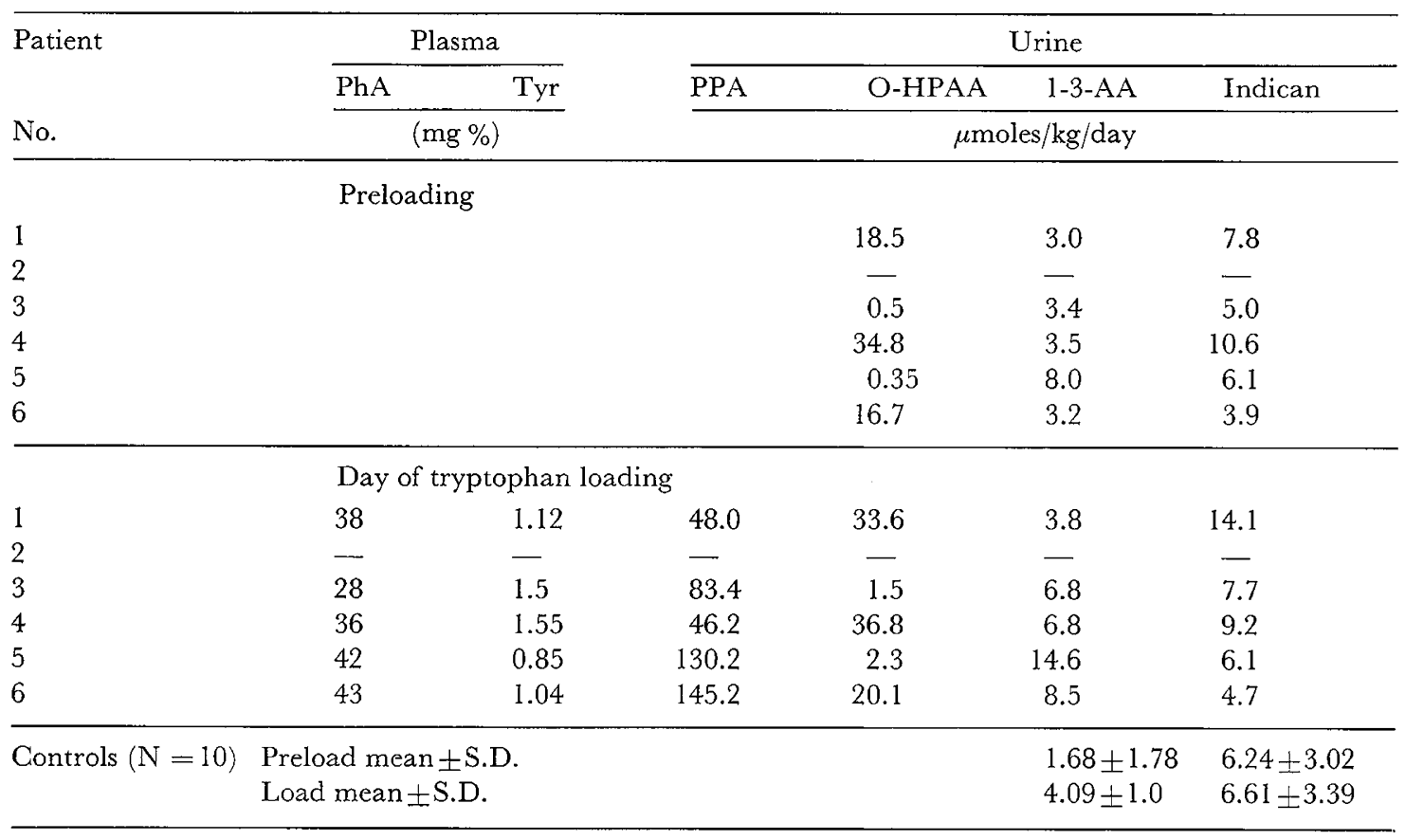

nine in serum were elevated. However, the failure of a tryptophan load to increase further the indican excretion in four of the subjects $(1,2,5$ and 6$)$ suggested that the tryptophan load was quite completely absorbed and that if indoles were formed by bacteria and absorbed, the capacity of the liver to form indican was already maximal [6].

When the subjects were placed on the low-phenylalanine diet, the excretion of 3-indoleacetic acid and indican during the preloading period became less in all of the patients. This suggested that less tryptophan was available in the bowel lumen for bacterial metabolism of tryptophan to indoles and that bacterial as well as endogenous production of 3-indoleacetic acid was decreased (table VI). As was observed on the general diet, tryptophan loading produced no change in indican excretion in four of the patients, Subjects 1 , 2, 5 and 6 (table VI). However, Subjects 3 and 4 showed a two- and four-fold increase respectively.

During the low-phenylalanine plus L-phenylalanine diet period, the excretion of 3-indoleacetic acid during the preloading period was again higher in four subjects, Subjects 3, 4, 5 and 6 than in normal subjects (table VII). Tryptophan loading produced a two-fold increase in four of the five subjects (table VII). The excretion of indican during the preloading and during the tryptophan loading period remained relatively un- changed and well within the range observed in the loaded control subjects $(6.61 \pm 3.39)$. The single exception was Subject 1.

These above observations suggested that when the serum phenylalanine concentration and the phenylpyruvic and ortho-hydroxyphenylacetic acid excretion are abnormally increased during administration of a general diet, some dietary tryptophan is not absorbed and remains in the bowel lumen shunted to the bacterial metabolic pool. Reduction of the serum phenylalanine concentration to within normal ranges and the consequent corresponding decreases in excretion of phenylpyruvic and ortho-hydroxyphenylacetic acids were associated with a reduced amount of tryptophan shunted to bacterial metabolism. However, when the subjects were on a low-phenylalanine diet containing sufficient added L-phenylalanine to increase the serum phenylalanine to the same values observed on the general diet, the excretion of indican remained essentially normal in four of the five subjects studied. 3indoleacetic acid excretion also remained about the same as observed on the low-phenylalanine diet alone. It should be recalled that while serum phenylalanine concentrations were greatly elevated, the excretion of phenylpyruvic and ortho-hydroxyphenylacetic acids was reduced on the low-phenylalanine plus L-phenylalanine diet. 


\section{Discussion}

The amount of kynurenine excreted following a load of L-tryptophan appears to be related to the amount of tryptophan pyrrolase activity in liver tissues [1]. As tryptophan is the specific substrate inducer of the enzyme, the amount of kynurenine and further metabolites produced will depend on the concentration of tryptophan that reaches the liver cells in an appropriate period of time [12]. Other factors are the rate of synthesis of the apotryptophan pyrrolase protein, which may be increased by hydrocortisone [11], and the availability of the heme coenzyme required for the formation of the active form of reduced holotryptophan pyrrolase [16].

The present studies indicate that inhibition of the oxidative pathway for tryptophan is present to a variable degree in untreated phenylketonurics. Certain subjects appear to have a marked inhibition of this pathway, and others, in spite of large production of phenylpyruvic acid and orthohydroxyphenylacetic acid, have essentially normal tryptophan oxidative metabolism reflected by the amount of tryptophankynurenine metabolites excreted following loading. The possibility that phenylpyruvic acid, ortho-hydroxyphenylacetic acid and indoles may have inhibited tryptophan pyrrolase activity in certain subjects may be supported by the in vivo observations of TADA and BESSMAN [31] and further in vitro observations by BessMAN [6]. In subjects in whom normal loading responses occurred, and despite the concomitant of production of large amounts of phenylpyruvic acid, there was no evidence of inhibition of tryptophan oxidation. In these subjects, other factors may be operative in overcoming the inhibition of tryptophan pyrrolase activity.

The normal or usually high tryptophan loading excretion responses for kynurenine metabolites in certain of the subjects, after being placed on the low-phenylalanine diet, suggested that some factor or factors present in the low-phenylalanine diet had a salutory effect on tryptophan pyrrolase activity, or that the above normal excretion response was an expression of a previously inhibited adaptive mechanism revealed only when the inhibitory factors were either partially or completely removed.

It was of interest that phenylpyruvic acid excretion in all of the subjects on the low-phenylalanine diet plus L-phenylalanine was about 30 to $50 \%$ of that found when they were consuming a general diet. This occurred even though the concentrations of phenylalanine in serum were equally elevated. This effect may be related to a competitive interplay between phenylalanine and tyrosine for the pyridoxine-dependent transamination systems required for the formation of phenyl- pyruvic acid from phenylalanine and the formation of para-hydroxyphenylpyruvic acid from tyrosine. It has been demonstrated in vitro that competition may occur between the phenylalanine-pyruvate transaminase and tyrosine-alpha-ketoglutarate transaminase enzyme [14]. It should also be noted here that the tyrosine-alpha-ketoglutarate transaminase level in the liver can be increased by hydrocortisone, while the phenylalanine-pyruvate transaminase cannot [20]. If inhibition is due to phenylpyruvic acid and orthohydroxyphenylacetic acid, a reduction in the amount of these acids would reduce the degree of inhibition of tryptophan pyrrolase activity and thus provide a partial explanation for increased tryptophan-kynurenine metabolite excretion observed following loading in the subjects when on the low-phenylalanine plus L-phenylalanine diet.

Ease of absorption of amino acids in the low-phenylalanine diet, in comparison with the availability from the general diet, may have been a factor which reduced the production of phenylpyruvic acid in the presence of high concentrations of phenylalanine in serum. The low-phenylalanine diet prepared by hydrolysis of casein contains about 7 to 10 g per 1000 calories of readily available glutamic acid. It has been demonstrated that L-glutamine, L-glutamate and L-asparagine given as a single oral load will reduce phenylpyruvic acid excretion in patients with phenylketonuria [23]. This effect may be due to a reversal of the following reaction: phenylalanine + alpha-ketoglutarate $\rightleftarrows$ phenylpyruvate + glutamate transamination. Hence two factors may have contributed to the reduced phenylpyruvic acid production: the reversal of the phenylalanine transamination reaction and increased production of endogenous hydrocortisone, which would increase the activity of tyrosine-alpha-ketoglutarate transaminase.

Other factors which may have contributed to the reduced metabolism of tryptophan observed in the present study are: 1. a limited absorption of tryptophan, which would provide less substrate for induction of tryptophan pyrrolase activity, and 2. an increased formation of inefficiently metabolized indole compounds, which also may contribute to the inhibition of tryptophan pyrrolase activity.

Interference with absorption of tryptophan by untreated patients with phenylketonuria may be of considerable significance while they are receiving a normal diet, one which contains approximately $0.4-0.5 \mathrm{~g}$ per 1000 calories of tryptophan. If dietary tryptophan is reduced, the loss of tryptophan shunted to bacterial metabolism may become significant. Approximate estimates of such losses in certain phenylketonuric subjects on the general diet indicated that as much as $26 \mu \mathrm{moles} / \mathrm{kg} / \mathrm{day}$ of 3 -indoleacetic acid plus indican was excreted (Subject 1, table I). Thus a daily excre- 
tion of $500 \mu$ moles of these two metabolites by a $19-\mathrm{kg}$ child would represent a loss of about $25 \%$ of the available dietary tryptophan (2000 $\mu$ moles). Losses of tryptophan by untreated phenylketonuric children may be even greater than those determined as free tryptophan found in the stool and unconjugated indoles found in the urine $[6,37]$.

In the present study, excretion of 3-indoleacetic acid and indican was 3 to 5 times above normal when the subjects were on the general diet. Tryptophan loading did not consistently or significantly increase the excretion of these metabolites in all subjects. In certain subjects, 3-indoleacetic acid excretion increased two- to three-fold (table V, Subject 5) and indican excretion increased two-fold during tryptophan loading (table $\mathrm{V}$, Subject 4). However, the percentage of the oral tryptophan load lost as 3-indoleacetic acid and indican in these subjects was probably not greater than 3 to $5 \%$ of the loads given, 9,120 and 16,620 $\mu$ moles tryptophan respectively. It would appear that decreased absorption of the tryptophan load did not significantly influence the amount of tryptophan available to the liver and could not, therefore, explain the decreased excretion of kynurenine and further oxidative metabolites.

The amount of indican and 3-indoleacetic acid excreted was less when the subjects were placed on the low-phenylalanine diet, and hence the presumed inhibitory influence of indoles on tryptophan pyrrolase activity may have been reduced. Further, the amount of indoles formed and excreted as indican in the subjects when placed on the low-phenylalanine plus Lphenylalanine diet was not greatly increased above the mean values observed for control children. Despite the high levels of phenylalanine in plasma, amounts equivalent to those found in subjects on a general diet, the amount of bacterially derived indole appeared to be less. This further reduced another potential inhibitor of tryptophan pyrrolase activity.

These studies provide no data concerning the role of catecholamine metabolism to the induction of tryptophan pyrrolase activity. The possibility exists that improvement in the limited synthesis $[25,34]$ of catecholamines $[7,26]$ or an increase in either tissue stores or blood concentration may also have indirectly contributed to the increased activity of tryptophan pyrrolase when the subjects were placed on a low-phenylalanine diet. This was also observed in certain subjects even when on a low-phenylalanine diet containing added L-phenylalanine. Epinephrine can evoke increased tryptophan pyrrolase activity by activating the pituitary-adrenal steroid-producing mechanism [18]. Indirectly, the improved and even enhanced activity of the tryptophan-kynurenine pathway may have resulted from increased production of hydrocortisone, a tryptophan pyrrolase inducer.
It is known that individual differences in the metabolism of phenylalanine to phenylpyruvic acid are present in phenylketonuria [13]. Rather marked variations in the production of ortho-hydroxy-phenylacetic acid unrelated to phenylpyruvic acid production have been noted [13]. The absorption of tryptophan from the bowel is probably also variable [37]. Differences in absorption of tryptophan would in turn produce differences in the amount of indoles derived from enteric bacterial metabolism. Individual differences in the absorption of dietary tyrosine may also exist, since competitive interaction in the absorption of tryptophan, phenylalanine and tyrosine has been suggested.

These above variables may be relevant to the differences in oxidative metabolism of tryptophan evoked by tryptophan loading observed in these phenylketonuric subjects. A summarization of the interrelation of these factors has been presented in figure 1 .

\section{Conclusions}

Decreased oxidation of tryptophan along the kynurenine degradation pathway was observed in certain untreated phenylketonuric children. This abnormality may be due to an inhibition of the activity of the tryptophan pyrrolase enzyme required for oxidation of tryptophan. Dietary induced decrease in the metabolites of phenylalanine and the bacterially derived metabolites of tryptophan resulted in improvement in the excretion of tryptophan-kynurenine metabolites, providing indirect evidence that inhibition of tryptophan pyrrolase activity may be associated with excess production of phenylketones and indoles. Certain phenylketonuric children failed to demonstrate limitation in oxidation of tryptophan in spite of the presence of high production of phenylpyruvic acid.

The different tryptophan loading responses observed in the phenylketonuric children on a general diet, a low-phenylalanine diet or on a low-phenylalanine diet plus L-phenylalanine suggests an individual variation in the degree of inhibition by phenylalanine and tryptophan metabolites on the tryptophan oxidative pathway and the presence of underlying adaptive mechanisms which may overcome the degree of inhibition.

These experiments were performed in a manner consistent with the rules and regulations of the University of Minnesota School of Medicine governing human experimentation and informed consent in force at the time these investigations were undertaken. 


\section{References and Notes}

1. Altman, K. and Greengard, O.: Correlation of kynurenine excretion with liver tryptophan pyrrolase levels in disease and hydrocortisone induction. J.clin. Invest. 45: 1527 (1966).

2. Anderson, J.A.; Fisch, R.; Miller, E. and DoeDEN, D.: Atypical phenylketonuric heterozygote. J.Pediat. 68: 351 (1966).

3. Armstrong, M.D. and Robinson, K.S.: On the excretion of indole derivatives in phenylketonuria. Arch. Biochem. Biophys. 52: 287 (1954).

4. Berendes, H.; Anderson, J.A.; Zregler, M.B. and Ruttenberg, D.: Disturbance in tryptophan metabolism in phenylketonuria. Amer.J.Dis. Child. 96: 430 (1958).

5. Bessman, S.P. and TADA, K.: On indicanuria in phenylketonuria. Metabolism 9: 377 (1960).

6. Bessman, S.P.: Some biochemical lessons to be learned from phenylketonuria. J.Pediat. 64: 828 (1964).

7. Boylen, J.B. and Quastel, J.H.: Effects of Lphenylalanine and sodium phenylpyruvate on the formation of adrenalin from L-tyrosine in adrenal medulla in vitro. Biochem.J. 80: 644 (1961).

8. Brown, R.R. and Price, J.M.: Quantitative studies on metabolites of tryptophan in urine of dog, cat, rat, and man. J. biol. Chem. 219: 985 (1956).

9. Brown, R.R.: The isolation and determination of urinary 3-hydroxykynurenine. J. biol. Chem. 227: 649 (1957).

10. Freedland, R.A.; Wadzinski, I.M. and WaisMAN, H.A.: The enzymatic hydroxylation of tryptophan. Biochem. Biophys. Res. Comm. 5: 94 (1961).

11. Greengard, O.; Smith, M.A. and Ace, G.: Relation of hydrocortisone and synthesis of ribonucleic acid to induced and developmental enzyme formations. J. biol. Ghem. 238: 1548 (1963).

12. Greengard, O. and Feigelson, P.: The activation and induction of rat liver tryptophan pyrrolase in vivo by its substrate. J. biol. Chem. 230: 158 (1961).

13. Jervis, G.A. and DrejzA, E.J.: Phenylketonuria: Blood levels of phenylpyruvic acid and ortho-hydroxyphenylacetic acid. Clin.chim. Acta 13: 435 (1966).

14. Kenney, F.T. and Kretchmer, N.: Hepatic metabolism of phenylalanine during development. J. clin. Invest. 38: 2185 (1959).

15. Knox, W.E. and Merler, A.H.: The conversion of tryptophan to kynurenine in liver. I. The coupled tryptophan peroxidase-oxidase system forming phenylkynurenine. J.biol. Chem. 187: 419 (1950).

16. KNox, W.E. and Piras, M.M.: A reinterpretation of the stabilization of tryptophan pyrrolase by its substrate. J. biol. Chem. 241: 764 (1966).
17. Knox, W.E.: The hormonal control of tryptophan pyrrolase in the rat. J. biol. Chem. 214: 307 (1955).

18. Knox, W.E.: Two mechanisms which increase in vivo the liver tryptophan pyrrolase activity; specific enzyme adaptation and stimulation of the pituitaryadrenal system. Brit.J. exp. Path. 32: 462 (1951).

19. KROPP, K. and LANG, K.: A colormetric method for quantitative determination of phenylpyruvic acid in urine. Klin.Wschr. 33: 482 (1955).

20. Lin, E. C. C. and KNox, N.E.: Specificity of adaptive response of tyrosine- $\alpha$-ketoglutarate transaminase. J. biol. Chem. 233: 1186 (1958).

21. MaCaman, N.W. and Robins, E.: Fluorimetric method for the determination of phenylalanine in serum. J.Lab. clin. Med. 59: 885 (1962).

22. Merklejohn, A.P. and Cohen, F.P.: The quantitative determination of indoxyl compounds in urine. J.Lab. clin. Med. 27: 949 (1942).

23. Meister, A.; Udenfriend, S. and Bessman, S.P.: Diminished phenylketonuria in phenylpyruvic oligophrenia after administration of L-glutamine, L-glutamate, L-asparagine. J. clin. Invest. 35: 619626 (1956).

24. Michael, A.F.; Drummond, K.N.; Doeden, D.; Anderson, J.A. and Good, R.A.: Tryptophan metabolism in man. J. clin. Invest. 43: 1730 (1964).

25. NAdLER, H.L. and Hsia, D.Y.: Epinephrine metabolism in phenylketonuria. Proc. Soc. exp. Biol. (N.Y.) 107: 721 (1961).

26. Nagatsu, T.; Levitt, M. and Udenfriend, S.: Tyrosine hydroxylase: The initial step in nonepinephrine synthesis. J. biol. Ghem. 239: 2910 (1964).

27. Pare, G.M.; Sandler, M. and Stacey, R.S.: 5-Hydroxytryptamine deficiency in phenylketonuria. Lancet $i$ : 551 (1957).

28. Renson, J.; Goodwin, F.; Weissbach, H. and UDENFRIEND, S.: Conversion of tryptophan to 5hydroxytryptophan by phenylalanine hydroxylase. Biochem. Biophys. Res. Comm. 6: 20 (1962).

29. SAtoh, K. and PrICE, J. M.: Fluorometric determinations of kynurenic and xanthurenic acid in human urine. J. biol. Chem. 230: 781 (1958).

30. Schreier, K. and Flaig, H.: Excretion of indolepyruvic acid in urine of normal subjects and patients with Folling's disease. Klin.Wschr. 34: 1213 (1956).

31. TADA, K. and Bessman, S.P.: Studies on tryptophan metabolism oligophrenia phenylpyruvica. Pediat.jap. 3: 41 (1960).

32. Tompsert, S.L.: 3-hydroxykynurenine in human urine. Clin. chim. Acta 5: 415 (1960).

33. WaAlkes, T.P. and Udenfriend, S.: A fluorometric method for estimation of tyrosine in plasma and tissues. J.Lab.clin. Med. 50: 733 (1957). 
34. Weil-Mahlerbe, H.: Blood adrenaline and intelligence, in biochemistry of developing nervous system (ed. WAELSEH, H.) 458: 466 (Academic Press New York 1955).

35. Weissbach, H.; King, W.; Sjoerdsma, A. and UDENFRIEND, S.: Formation of indole-3-acetic and tryptamine in animals. J. biol. Chem. 234:81 (1959).

36. Williams, C.M. and Sweeley, C.C.: Gas chromatography of urinary aromatic acids. Biochemical applications of gas chromatography (ed. SzyManski, H.A.) (Plenum Press, New York 1964).
37. Yarbro, M. and Anderson, J.A.: L-tryptophan metabolism in phenylketonuria. J.Pediat. 68: 895 (1966).

38. Supported in part by grants from the McClure Metabolic Research Fund, The Minnesota Association for Retarded Children, and The Graduate School Research Fund of the University of Minnesota.

39. Anderson, J.A., M.D., Professor and Head, Department of Pediatrics, University of Minnesota Medical School, Minneapolis, Minn. 55455 (USA). 